\title{
A!
}

This is an electronic reprint of the original article.

This reprint may differ from the original in pagination and typographic detail.

Kivisaari, Pyry; Partanen, Mikko; Oksanen, Jani

\section{Optical admittance method for light-matter interaction in lossy planar resonators}

Published in:

Physical Review E

DOI:

10.1103/PhysRevE.98.063304

Published: 04/12/2018

Document Version

Publisher's PDF, also known as Version of record

Please cite the original version:

Kivisaari, P., Partanen, M., \& Oksanen, J. (2018). Optical admittance method for light-matter interaction in lossy planar resonators. Physical Review E, 98(6), 1-13. [063304]. https://doi.org/10.1103/PhysRevE.98.063304

This material is protected by copyright and other intellectual property rights, and duplication or sale of all or part of any of the repository collections is not permitted, except that material may be duplicated by you for your research use or educational purposes in electronic or print form. You must obtain permission for any other use. Electronic or print copies may not be offered, whether for sale or otherwise to anyone who is not an authorised user. 


\title{
Optical admittance method for light-matter interaction in lossy planar resonators
}

\author{
Pyry Kivisaari, ${ }^{*}$ Mikko Partanen, and Jani Oksanen \\ Engineered Nanosystems Group, Aalto University, P.O. Box 13500, FI-00076 Aalto, Finland
}

(Received 22 August 2018; published 4 December 2018)

\begin{abstract}
Advanced optoelectronic simulation models are needed to study and optimize emerging photonic devices such as thin-film solar cells, lasers, and light-emitting diodes (LEDs). In particular, better tools are required for self-consistent modeling of coupled electrical and optical systems. The recently introduced quantized fluctuational electrodynamics (QFED) and the associated interference-exact radiative transfer equations have been developed for this purpose, but their use is in part complicated by the need to calculate the full dyadic Green's functions. To make QFED and the underlying physical quantities more accessible for new device studies, we introduce a directly usable method where Green's functions are obtained through optical admittances. The optical admittances can be solved analytically for piecewise-homogeneous layer structures and selected gradedindex profiles, and numerically for arbitrary position-dependent refractive index profiles using well-known techniques. The solutions enable direct construction of the dyadic Green's functions and all the related optical quantities. To give examples of the general applicability of the method, we calculate the local and nonlocal optical densities of states for selected devices, including GaN-based flip-chip LEDs and vertical-cavity surface-emitting lasers. Using only the rather simple framework presented in this paper, one can analyze energy transport in a wide range of planar photonic devices accurately without additional difficulties or inputs from external solvers.
\end{abstract}

DOI: 10.1103/PhysRevE.98.063304

\section{INTRODUCTION}

Advances in optoelectronics during the last few decades have had major impacts in society, with high-efficiency solidstate lighting and solar photovoltaics standing out as prime examples $[1,2]$. As conventional light-emitting diodes (LEDs) and photovoltaic cells are already rapidly gaining ground in general lighting and energy production, new materials and nanostructures are being studied and engineered into use in more sophisticated optoelectronics applications not yet available on the market. Examples of these include, e.g., electroluminescent cooling [3,4], optical on-chip communication using nanostructure-based optical interconnects [5,6], photocatalytic solar fuel production [7], solar cells made of unconventional earth-abundant materials [8], new light emitters and absorbers targeted for biomedical applications [9,10], and diverse nanowire-based devices [11]. Studying and developing these applications requires sophisticated yet sufficiently accessible and insightful full-device modeling tools where nanoscale simulation of optical, electrical, and thermal processes is fully integrated.

One of the most important missing pieces in photonic nanodevice modeling is the full coupling of optical and electrical processes. Essentially this means that the emission, transport, and absorption of photons would be self-consistently accounted for in the carrier dynamics simulation. This is typically done only in specific cases, with or without wave-optical effects. For example, Wang et al. treated photon recycling with a ray-tracing model in GaAs solar cells [12], Durbin and Gray used a Beer-Lambert expression to account for photon

*pyry.kivisaari@aalto.fi recycling [13], and in a previous work we used fluctuational electrodynamics (FED) to model emission enhancement with plasmonic gratings [14]. On the other hand, there are several works where the topic has been explored in nanostructures with fairly specialized and computationally heavy frameworks [15-17]. Our aim, in contrast to previous works, is to construct and present an accurate yet straightforward method for modeling the emission, transport, and absorption of photons in arbitrary planar photonic devices while fully accounting for the pertinent interference and other wave-optical effects.

To simplify the fundamental models of light-matter interaction in planar semiconductor devices, in this paper we show how the optical densities of states needed in FED and the more advanced quantized fluctuational electrodynamics (QFED) [18-20] can be calculated directly by expressing the electromagnetic Green's functions with optical admittances $[21,22]$. Typically the calculation of Green's functions in planar structures requires finding two independent eigenmodes of the homogeneous Helmholtz equations (such as the propagating modes incident on the structure from left or right as illustrated in Fig. 1). These modes can be calculated using transfer matrices, numerical methods, or other established techniques, and such approaches have been widely used, e.g., in the prior research on the emission and absorption of distributed feedback (DFB) lasers [23-25]. However, using these techniques can be cumbersome due to the need to match the eigenmodes with the free-space Green's function at the source location to fix the unknown field amplitudes [26]. On the other hand, impedances and admittances and the closely related Smith charts are frequently used in transmission line theory to solve wave equations and maximize signal transmission. Considering their apparent convenience for solving wave equations, it is even surprising that admittances are not used 


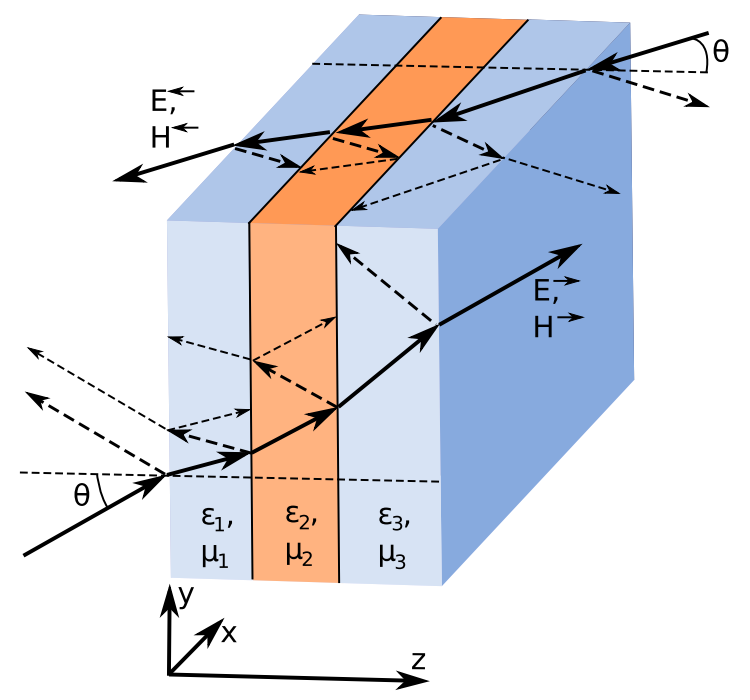

FIG. 1. Schematic illustration of the general layer structure modeled using the optical admittance method. The figure also shows ray-optical illustrations of principally right- and left-propagating optical modes $E^{\rightarrow}, H^{\rightarrow}$ and $E^{\leftarrow}, H^{\leftarrow}$. The fields generally have components propagating in both forward and backward directions, apart from the final half-spaces where the modes exit the structure.

more frequently in wave optics. Here we show that the easily evaluated optical admittances provide an out-of-the-box tool to express the dyadic Green's functions and thereby the local and nonlocal densities of states (LDOS and NLDOS) directly without having to formally solve the electromagnetic fields. Having such a direct tool to accurately couple together carrier and photon dynamics makes it notably more convenient than previously to perform all-optoelectronic modeling studies of planar device structures. This is expected to enable both increasing the performance of existing applications and creating new device functionalities.

Even if the optical and electrical properties of planar structures have been studied extensively, often the focus is mostly on only one of them while simplifying approximations are made for the other. Having a directly workable, fully self-consistent model for both electrical and optical transport is therefore expected to enable various interesting studies. Examples include optimizing both the carrier dynamics and photon recycling in heterostructure thin-film solar cells made of traditional or emerging materials, and maximizing the net photon absorption and photocarrier collection in electroluminescent cooling devices. Moreover, using perturbative or other approximative techniques [14], simulation models for planar structures can be generalized, even for devices containing nanostructures, such as optical nanoresonators and optical antennas.

\section{THEORY}

In this section, we review the physical significance of the electromagnetic Green's functions both for classical and quantized FED and show how they can be calculated using the optical admittances. More precisely, Sec. II A presents the homogeneous and inhomogeneous Maxwell's equations in planar structures and briefly reviews how scalar Green's functions can be used to calculate the fields resulting from electric and magnetic sources. Sections II B-II C summarize the optical admittance method and show how the scalar Green's functions are obtained from the optical admittances for an arbitrary propagation angle. Finally, Secs. IID-II E generalize the scalar Green's functions to their vectorial (or dyadic) forms and show how the optical admittances can be used to directly obtain all the quantities needed in classical and quantized FED, as well as in the interference-exact radiative transfer model which can be readily coupled with carrier dynamics models. We note here that in the end, all these quantities are obtained without solving the electromagnetic fields formally, e.g., with external solvers.

\section{A. Homogeneous and inhomogeneous Maxwell's equations}

Emission, propagation, and absorption of photons is described by Maxwell's equations, which dictate how the Fourier components (in frequency domain) of the electric field $\mathbf{E}$, magnetic field $\mathbf{H}$, electric flux density $\mathbf{D}$, and magnetic flux density $\mathbf{B}$ relate to the Fourier components of the electric charge density $\rho_{f}$ and the polarization and magnetization current densities $\mathbf{J}_{e}$ and $\mathbf{J}_{m}$. For positive angular frequencies $\omega$ giving the time dependence of the fields as $\exp (-i \omega t)$, Maxwell's equations in frequency domain are given by

$$
\begin{gathered}
\nabla \cdot \mathbf{D}=\rho_{f}, \\
\nabla \cdot \mathbf{B}=0, \\
\nabla \times \mathbf{E}=-\mathbf{J}_{m}+i \omega \mu_{0} \mu \mathbf{H}, \\
\nabla \times \mathbf{H}=\mathbf{J}_{e}-i \omega \varepsilon_{0} \varepsilon \mathbf{E},
\end{gathered}
$$

where $\varepsilon_{0}$ and $\mu_{0}$ are the permittivity and permeability of vacuum, and the relative permittivity $\varepsilon$ and relative permeability $\mu$ describe the polarization and magnetization that depend linearly on the electric and magnetic fields. The generally nonlinear polarization and magnetization currents are given by $\mathbf{J}_{e}=\mathbf{J}_{f}-i \omega \delta \mathbf{P}$ and $\mathbf{J}_{m}=-i \omega \mu_{0} \delta \mathbf{M}$, where $\mathbf{J}_{f}$ is a linear free electric current density term and $\delta \mathbf{P}$ and $\delta \mathbf{M}$ are the polarization and magnetization that do not follow a linear dependence of the fields. In both classical and quantized FED, the thermal fluctuations that act as sources of the fields are included as noise currents in $\mathbf{J}_{e}$ and $\mathbf{J}_{m}[18,27]$.

In this paper we focus on planar devices illustrated in Fig. 1, where the material only changes in the $z$ direction. Such structures have perfect symmetry in the $x y$ plane, and the full electromagnetic mode space can be described using the well-known transverse electric (TE) and transverse magnetic (TM) modes defined by their plane of propagation, so that TE (TM) modes have nonzero electric (magnetic) field only in the direction $\mathbf{u}_{1}$ in the $x y$ plane perpendicular to the plane of propagation. To define an alternative orthogonal set of Cartesian coordinates associated with the plane of propagation, we define a device plane unit vector $\mathbf{u}_{K}$ through the relation $\mathbf{u}_{1}=\mathbf{u}_{K} \times \mathbf{u}_{z}$, so that $\mathbf{u}_{K}$ is the projection of the wave vector $\mathbf{k}$ onto the $x y$ plane perpendicular to $\mathbf{u}_{1}$, and $\mathbf{u}_{z}$ is the $z$ direction of Fig. 1. The coordinates corresponding to $\mathbf{u}_{1}, \mathbf{u}_{K}$, and $\mathbf{u}_{z}$ are then chosen as $u, v$, and $z$, respectively. Furthermore, we assume here for simplicity that the permittivity and 
permeability of the material do not depend on the polarization of light, but this approximation can be removed.

Due to the symmetry of the system, Maxwell's equations can be further simplified into the customary scalar nonhomogeneous Helmholtz equations. The Helmholtz equation for the TE modes can be derived for any propagation plane by dividing Eq. (3) by $\mu(z)$, taking the curl, and making use of vector calculus and Eq. (4). This leads to

$$
\begin{aligned}
& \frac{1}{\mu} \frac{d^{2}}{d v^{2}} E(v, z)+\frac{d}{d z}\left(\frac{1}{\mu} \frac{d E(v, z)}{d z}\right)+k_{0}^{2} \varepsilon E(v, z) \\
& \quad=J_{\mathrm{eff}}^{T E}(v, z),
\end{aligned}
$$

where $E$ is the magnitude of $\mathbf{E}$ along $\mathbf{u}_{1}, k_{0}=\omega / c$ ( $c$ being the speed of light in vacuum), and $J_{\text {eff }}^{T E}(v, z)=\mathbf{u}_{1}$. $\left[-i \omega \mu_{0} \mathbf{J}_{e}+\nabla \times\left(\frac{1}{\mu} \mathbf{J}_{m}\right)\right]$, so that only the source components normal to the plane of propagation generate the TE field. In general, however, the $\mathbf{J}_{e}$ and $\nabla \times\left(\mathbf{J}_{m} / \mu\right)$ vectors have three independent components (e.g., $x, y, z$ ), with two of them $(x, y)$ contributing to $J_{\text {eff }}^{T E}$, and it will be necessary to account for the $x$ and $y$ components separately if one is interested in the field correlations between fields with different planes of propagation. To deal with the derivatives in the $x y$ plane, we write the Fourier transform of $E$ and $J_{\text {eff }}^{T E}$ as

$$
E(v, z)=\frac{1}{(2 \pi)^{2}} \int_{K} E(z, K) \exp (i K v) d K,
$$

where $K=\mathbf{u}_{K} \cdot \mathbf{k}$ is the in-plane component of the wave vector $\mathbf{k}$, so that the full wave vector is written as $\mathbf{k}=$ $\mathbf{K}+k_{z} \mathbf{u}_{z}=K \mathbf{u}_{K}+k_{z} \mathbf{u}_{z}$, with $k_{z}$ the $z$ component of $\mathbf{k}$. The Fourier transform of $J_{\text {eff }}^{T E}$ is written similarly with its Fourier coefficients denoted as $J_{\text {eff }}(z, K)$. By substituting Eq. (6) to Eq. (5) and requiring it to hold separately for each $K$, we get the Helmholtz equation for TE modes as

$$
\begin{aligned}
& \frac{d}{d z}\left(\frac{1}{\mu} \frac{d E(z, K)}{d z}\right)+k_{0}^{2}\left(\varepsilon-\frac{1}{\mu} \frac{K^{2}}{k_{0}^{2}}\right) E(z, K) \\
& \quad=J_{\text {eff }}^{T E}(z, K) .
\end{aligned}
$$

For TM modes, we use the same symmetry arguments as for TE and choose the magnetic field as $\mathbf{H}=H \mathbf{u}_{1}$. The Helmholtz equation for $H$ can then be derived in a similar way, resulting in

$$
\begin{aligned}
& \frac{d}{d z}\left(\frac{1}{\varepsilon} \frac{d H(z, K)}{d z}\right)+k_{0}^{2}\left(\mu-\frac{1}{\varepsilon} \frac{K^{2}}{k_{0}^{2}}\right) H(z, K) \\
& \quad=J_{\mathrm{eff}}^{T M}(z, K),
\end{aligned}
$$

where $J_{\text {eff }}^{T M}(z, K)$ is the Fourier component of $J_{\text {eff }}^{T M}(v, z)=$ $\mathbf{u}_{1} \cdot\left[-i \omega \varepsilon_{0} \mathbf{J}_{m}-\nabla \times\left(\frac{1}{\varepsilon} \mathbf{J}_{e}\right)\right]$.

Both classical FED and QFED are based on writing $\mathbf{J}_{e}$ and $\mathbf{J}_{m}$ using the fluctuation-dissipation theorem (FDT). With position-dependent $\mathbf{J}_{e}$ and $\mathbf{J}_{m}$ from the FDT, solving Eqs. (7) and (8) is dramatically simplified by using the electromagnetic Green's functions, which describe how a plane source located at $z=z_{0}$ generates an electromagnetic field in the surrounding space. In other words, the Green's function for TE modes
$G^{T E}\left(z, z_{0}, k_{0}, K\right)$ satisfies

$$
\frac{d}{d z}\left(\frac{1}{\mu} \frac{d G^{T E}}{d z}\right)+k_{0}^{2}\left(\varepsilon-\frac{1}{\mu} \frac{K^{2}}{k_{0}^{2}}\right) G^{T E}=-\delta\left(z-z_{0}\right),
$$

where $\delta$ is the Dirac $\delta$ function. The Green's function $G^{T M}\left(z, z_{0}, k_{0}, K\right)$ for TM modes satisfies a corresponding modification of Eq. (8). $G^{T E}$ and $G^{T M}$ are the so-called scalar Green's functions, which are generalized to their dyadic forms later. Note that Eq. (9) and the corresponding TM equation essentially describe how a plane source located at $z=z_{0}$ generates fields that propagate according to the homogeneous Helmholtz equations. The homogeneous Helmholtz equations are Eqs. (7) and (8) with their right-hand sides set to zero, and therefore they describe the propagation and attenuation of electromagnetic fields within the layer structure according to linear optics. When the scalar Green's functions are known, the TE electric field at $z$ due to arbitrarily distributed $\mathbf{J}_{e}$ and $\mathbf{J}_{m}$ is obtained as

$$
E\left(z, k_{0}, K\right)=\int_{-\infty}^{\infty} G^{T E}\left(z, z_{0}, k_{0}, K\right) J_{\text {eff }}^{T E}\left(z_{0}, k_{0}, K\right) d z_{0} .
$$

The TM magnetic field is obtained from $\mathbf{J}_{e}$ and $\mathbf{J}_{m}$ using a corresponding integral.

\section{B. The optical admittance method}

As detailed in the previous section, Green's functions describe how the electromagnetic field generated by a point source propagates in the structure. Solving the Green's functions therefore typically requires solving the homogeneous Helmholtz equations. Here we repeat briefly the derivation of optical admittances from $[21,22]$ and show that they provide a rather convenient way to do this for an arbitrary propagation angle. Later in Secs. II C-II D we show how the optical admittances can be used to express $G^{T E}$ and $G^{T M}$ and eventually the full dyadic Green's functions and optical densities of states required to model the light-matter interactions in the classical and quantized FED.

Using the field components parallel to the interfaces, we define the optical admittances as [21,22]

$$
\begin{gathered}
\gamma^{T E}(z)=-\sqrt{\frac{\mu_{0}}{\varepsilon_{0}}} \frac{H_{\|}(z)}{E(z)}, \\
\gamma^{T M}(z)=\sqrt{\frac{\varepsilon_{0}}{\mu_{0}}} \frac{E_{\|}(z)}{H(z)},
\end{gathered}
$$

where $H_{\|}=\mathbf{u}_{K} \cdot \mathbf{H}^{T E}$ is the magnetic field in the $x y$ plane in the TE modes, and $E_{\|}=\mathbf{u}_{K} \cdot \mathbf{E}^{T M}$ is the corresponding electric field in the TM modes. The admittances are therefore written for each $k_{0}$ and $K$, but these dependencies are omitted in the arguments here for brevity. Note that we have defined $\gamma^{T M}$ in Eq. (12) as the inverse of the TM admittance of Ref. [21] to simplify the rest of the equations in this paper. Strictly speaking, $\gamma^{T M}$ should therefore be called impedance rather than admittance. With $\sigma \in\{T E, T M\}$ representing the polarization, the optical admittances $\gamma^{\sigma}$ defined in Eqs. (11) and (12) must be continuous over interfaces between different 
layers due to the general continuity requirements of tangential fields. From Maxwell's equations (1)-(4), the remaining fields needed in Eqs. (11) and (12) are obtained as $H_{\|}=$ $-i /\left(\omega \mu_{0} \mu\right) d E / d z$ and $E_{\|}=i /\left(\omega \varepsilon_{0} \varepsilon\right) d H / d z$, when the current terms are set to zero as imposed by the homogeneity condition. Substituting these into Eqs. (11) and (12) and solving $E$ and $H$ gives

$$
\begin{aligned}
& E(z)=E_{0} \exp \left(-i k_{0} \int_{z_{0}}^{z} \mu \gamma^{T E}\left(z^{\prime}\right) d z^{\prime}\right), \\
& H(z)=H_{0} \exp \left(-i k_{0} \int_{z_{0}}^{z} \varepsilon \gamma^{T M}\left(z^{\prime}\right) d z^{\prime}\right),
\end{aligned}
$$

where $E_{0}$ and $H_{0}$ are arbitrary initial values at $z=z_{0}$, which cancel out from the Green's functions, as will be shown later.

We note that the modes described by Eqs. (13) and (14) account for all refraction, reflection, interference, and absorption effects within the structure (but not emission), and therefore they generally include both forward- and backwardpropagating wave forms. (See Fig. 1 for ray-optics illustrations of the two independent modes.) Substituting Eqs. (13) and (14) into the homogeneous forms of Eqs. (7) and (8) results in first-order differential equations for the optical admittances given by $[21,22]$

$$
\begin{aligned}
& \frac{d \gamma^{T E}(z)}{d z}=i k_{0} \mu\left[\gamma^{T E}(z)^{2}-\kappa^{T E}(z)^{2}\right], \\
& \frac{d \gamma^{T M}(z)}{d z}=i k_{0} \varepsilon\left[\gamma^{T M}(z)^{2}-\kappa^{T M}(z)^{2}\right],
\end{aligned}
$$

where $\kappa^{\sigma}$ are scaled propagation constants defined as

$$
\begin{gathered}
\kappa^{T E}(z)=-\frac{\sqrt{\varepsilon \mu-K^{2} / k_{0}^{2}}}{\mu}, \\
\kappa^{T M}(z)=-\frac{\sqrt{\varepsilon \mu-K^{2} / k_{0}^{2}}}{\varepsilon} .
\end{gathered}
$$

The boundary conditions for Eqs. (15) and (16) can be chosen so that the solutions represent modes incident from the left and exiting to the right and modes incident from the right and exiting to the left side of the structure. On the right (left) side of the structure, modes exiting to the right (left) exhibit only a single wave form with $E, H \sim \exp \left(i k_{z} z\right)(E, H \sim$ $\left.\exp \left(-i k_{z} z\right)\right)$. To match this boundary condition with Eqs. (13) and (14), the optical admittances for modes exiting to the right (left) must be equal to the scaled propagation constants of Eqs. (17) and (18) in the right (left) half-space. In other parts of the structure, the admittances are governed by Eqs. (15) and (16), which can be solved analytically or numerically, requiring that $\gamma^{\sigma}$ is continuous over all boundaries.

For a layer with constant $\varepsilon$ and $\mu$, the optical admittance can be expressed analytically as

$$
\gamma^{\sigma}(\Delta z)=-\kappa^{\sigma} \tanh \left[i k_{z} \Delta z+\operatorname{arctanh}\left(-\frac{\gamma_{b}^{\sigma}}{\kappa^{\sigma}}\right)\right]
$$

where $\Delta z=z_{b}-z$ (rightward modes) and $\Delta z=z-z_{b}$ (leftward modes), $z_{b}$ is the $z$ coordinate of the interface where the mode exits the layer, and $\gamma_{b}^{\sigma}$ is the initial value of $\gamma^{\sigma}$ at $z_{b}$. For a structure with piecewise constant $\varepsilon$ and $\mu$, the full solution can be easily constructed by starting from the final outer boundary where the admittances match the propagation constants of Eqs. (17) and (18). One then writes the admittances subsequently for all the layers using Eq. (19) and requiring continuity at each interface. On the other hand, if there are graded layers in the structure, the admittances in those layers have to be solved numerically or by using analytical solutions that apply for specific graded-index profiles [22].

Solving the optical admittances $\gamma_{r}^{\sigma}$ and $\gamma_{l}^{\sigma}$ for modes exiting to the right and left, respectively, corresponds to solving two independent eigenmodes of the homogeneous Maxwell's equations. However, no formal determination of the optical field profiles nor simultaneous accounting of the left- and right-propagating fields is needed to gain access to, e.g., the Green's functions. Note that the process formulated in this section is very similar to a typical calculation of signal propagation within a transmission line and the related Smith's charts, where the position-dependent current and voltage are represented by equations similar to Eqs. (7) and (8), and position-dependent impedances and admittances are defined as their ratio.

\section{Scalar Green's functions from optical admittances}

Having derived the optical admittances, the scalar Green's functions $G^{T E}$ and $G^{T M}$ can be obtained by using a mapping between the homogeneous fields and the scalar Green's functions as presented for normal incidence by Di Stefano et al. [28]. The mapping is based on a more general framework for solving Green's functions (see, e.g., Ref. [29]), where the Green's function outside the source point is first constructed from the homogeneous solutions exiting to the right and left, and the solution is normalized by a Wronskian to satisfy the differential equation also at the source point. Using the sign conventions of Ref. [18] and generalizing for arbitrary $\varepsilon, \mu$, and $K$, this results in scalar Green's functions given by

$$
G^{T E}\left(z, z_{0}, k_{0}, K\right)=\frac{-\mu(z)}{W^{T E}\left(k_{0}, K\right)}\left[E^{\rightarrow}\left(z, k_{0}, K\right) E^{\leftarrow}\left(z_{0}, k_{0}, K\right) \Theta\left(z-z_{0}\right)+E^{\leftarrow}\left(z, k_{0}, K\right) E^{\rightarrow}\left(z_{0}, k_{0}, K\right) \Theta\left(z_{0}-z\right)\right]
$$

and

$$
G^{T M}\left(z, z_{0}, k_{0}, K\right)=\frac{-\varepsilon(z)}{W^{T M}\left(k_{0}, K\right)}\left[H^{\rightarrow}\left(z, k_{0}, K\right) H^{\leftarrow}\left(z_{0}, k_{0}, K\right) \Theta\left(z-z_{0}\right)+H^{\leftarrow}\left(z, k_{0}, K\right) H^{\rightarrow}\left(z_{0}, k_{0}, K\right) \Theta\left(z_{0}-z\right)\right],
$$

where $z_{0}$ is the source point coordinate, $E^{\rightarrow}, E^{\leftarrow}$ and $H^{\rightarrow}, H^{\leftarrow}$ are the electric and magnetic fields of the modes exiting to the right and left side of the structure for TE and TM, and $\Theta$ is the unit step function. Finally, $W^{\sigma}\left(k_{0}, K\right)$ in Eqs. (20) and (21) is 
the Wronskian defined for TE as

$$
W^{T E}\left(k_{0}, K\right)=E^{\leftarrow}\left(z, k_{0}, K\right) \frac{\partial}{\partial z} E^{\rightarrow}\left(z, k_{0}, K\right)-E^{\rightarrow}\left(z, k_{0}, K\right) \frac{\partial}{\partial z} E^{\leftarrow}\left(z, k_{0}, K\right),
$$

and similarly for TM by replacing $E$ with $H$.

The scalar Green's functions can now be expressed with the optical admittances by substituting Eqs. (13) and (14) into Eqs. (20) and (21), resulting in

$$
\begin{aligned}
& G^{T E}\left(z, z_{0}, k_{0}, K\right)=\frac{-i}{k_{0}} \frac{1}{\gamma_{r}^{T E}(z)+\gamma_{l}^{T E}(z)}\left[\exp \left(-i k_{0} \int_{z_{0}}^{z} \varepsilon \gamma_{l}^{T E} d z^{\prime}\right) \Theta\left(z-z_{0}\right)+\exp \left(-i k_{0} \int_{z}^{z_{0}} \varepsilon \gamma_{r}^{T E} d z^{\prime}\right) \Theta\left(z_{0}-z\right)\right] \\
& G^{T M}\left(z, z_{0}, k_{0}, K\right)=\frac{-i}{k_{0}} \frac{1}{\gamma_{r}^{T M}(z)+\gamma_{l}^{T M}(z)}\left[\exp \left(-i k_{0} \int_{z_{0}}^{z} \varepsilon \gamma_{l}^{T M} d z^{\prime}\right) \Theta\left(z-z_{0}\right)+\exp \left(-i k_{0} \int_{z}^{z_{0}} \varepsilon \gamma_{r}^{T M} d z^{\prime}\right) \Theta\left(z_{0}-z\right)\right] .
\end{aligned}
$$

Looking at Eqs. (23) and (24), one can directly see that at the limit $z \rightarrow z_{0}$, the integral terms vanish and the scalar Green's functions are simply given by the prefactors of the equations.

\section{Dyadic Green's functions and densities of states}

Using Eq. (10) and the corresponding equation for the TM magnetic field, the scalar Green's functions $G^{T E}$ and $G^{T M}$ can be directly used to evaluate how a current source or its curl [cf. Eqs. (7) and (8)] excites the electric field of a TE mode or the magnetic field of a TM mode. The transverse electric or magnetic field then provides direct access to the corresponding magnetic and electric field components through the curls in Eqs. (3) and (4). However, to more conveniently keep track of the fields and source orientations, one typically makes use of the fundamental vector calculus theorems and includes all the derivatives and directions in the so-called vectorial (or dyadic) Green's function. Below we loosely follow Refs. [19] and [20] and review how to break down the full dyadic Green's function to its different components.

The dyadic Green's function can be understood by considering how an arbitrary point source creates electric and magnetic fields with a given $\mathbf{K}$ vector. For simplicity, we construct the dyadic in the basis defined by the vectors $\mathbf{u}_{1}, \mathbf{u}_{K}$, and $\mathbf{u}_{z}$ so that the current terms generating the fields are also projected or rotated to this basis. As an example, the components of the electric field resulting from the electric current source components along the basis directions $\mathbf{u}_{1}, \mathbf{u}_{K}, \mathbf{u}_{z}$ are then given by

$$
E_{i}\left(z, k_{0}, K\right)=i \omega \mu_{0} \int_{-\infty}^{\infty} \sum_{j} g_{e e}^{i j}\left(z, z_{0}, k_{0}, K\right) J_{e, j}\left(z_{0}, k_{0}, K\right) d z_{0},
$$

where $i, j \in\{1, K, z\}$. Electric sources in the $\mathbf{u}_{1}$ direction create only TE electric fields along $\mathbf{u}_{1}$ as described by the components $g_{e e}^{11}=G^{T E}$ and $g_{e e}^{K 1}=g_{e e}^{z 1}=0$ of the dyadic Green's function. Electric sources in the $\mathbf{u}_{K}$ and $\mathbf{u}_{z}$ direction correspondingly create only TM modes, which have electric fields both in the $\mathbf{u}_{K}$ and $\mathbf{u}_{z}$ directions, and these fields are described by the dyadic components $g_{e e}^{K K}, g_{e e}^{z K}$, and $g_{e e}^{1 K}=0$, and $g_{e e}^{K z}, g_{e e}^{z z}$, and $g_{e e}^{1 z}=0$, respectively, with the associated formulas given in Appendix A. Accordingly, one can understand the magnetic Green's functions $g_{m m}^{i j}$ describing how magnetic sources create magnetic fields, and the exchange Green's functions $g_{m e}^{i j}$ and $g_{e m}^{i j}$, which describe how electric sources create magnetic fields and magnetic sources create electric fields, respectively. All the dyadic Green's function components can be derived from the scalar Green's functions using Maxwell's equations and Green's identities as shown in Ref. [20], and we have included them as functions of the optical admittances in Appendix A.

Writing the dyadic Green's function with optical admittances allows expressing all the LDOS and NLDOS required in the classical and quantized FED. In vacuum, the electric and magnetic LDOS are proportional to the trace of the imaginary parts of the dyadic Green's functions [30,31]. In a lossy medium, on the other hand, the LDOS can be calculated as the integral of the NLDOS. In Ref. [19], we showed that by using the dyadic Green's function components introduced above and setting $z \rightarrow z_{0}$, the resulting electric and magnetic LDOS are obtained as $[19,20]$

$$
\begin{array}{r}
\rho_{e}\left(z, k_{0}, K\right)=\frac{k_{0}}{4 \pi^{3} c} \Im\left\{g_{e e}^{11}+g_{e e}^{K K}+\frac{\varepsilon^{2}}{|\varepsilon|^{2}} g_{e e}^{z z}\right\}, \\
\rho_{m}\left(z, k_{0}, K\right)=\frac{k_{0}}{4 \pi^{3} c} \Im\left\{g_{m m}^{11}+g_{m m}^{K K}+\frac{\mu^{2}}{|\mu|^{2}} g_{m m}^{z z}\right\} .
\end{array}
$$

Note that in the limit of small losses, $\varepsilon^{2} \approx|\varepsilon|^{2}$ and $\mu^{2} \approx|\mu|^{2}$, and these equations correspond to the above-mentioned conventional LDOS formula for vacuum. Also, the well-known divergence of the pointwise LDOS in lossy media [30,31] only appears after the inverse Fourier transform integration over large $K$ values corresponding to evanescent waves. With the above 
expressions, the LDOS can now be obtained using the optical admittances directly as a simple expression,

$$
\rho_{e}\left(z, K, k_{0}\right)=\frac{-1}{4 \pi^{3} c} \Re\left\{\left(\frac{1}{\gamma_{r}^{T E}+\gamma_{l}^{T E}}+\frac{\gamma_{r}^{T M} \gamma_{l}^{T M}}{\gamma_{r}^{T M}+\gamma_{l}^{T M}}+\frac{\left(K / k_{0}\right)^{2}}{|\varepsilon|^{2}} \frac{1}{\gamma_{r}^{T M}+\gamma_{l}^{T M}}\right)\right\}
$$

for the electric field and

$$
\rho_{m}\left(z, K, k_{0}\right)=\frac{-1}{4 \pi^{3} c} \Re\left\{\left(\frac{1}{\gamma_{r}^{T M}+\gamma_{l}^{T M}}+\frac{\gamma_{r}^{T E} \gamma_{l}^{T E}}{\gamma_{r}^{T E}+\gamma_{l}^{T E}}+\frac{\left(K / k_{0}\right)^{2}}{|\mu|^{2}} \frac{1}{\gamma_{r}^{T E}+\gamma_{l}^{T E}}\right)\right\}
$$

for the magnetic field. The total electromagnetic LDOS for the energy density can be calculated from these as $\rho_{\text {tot }}=1 / 2\left(|\varepsilon| \rho_{e}+\right.$ $\left.|\mu| \rho_{m}\right)$.

For completeness, the electric NLDOS can be calculated from the dyadic Green's function components as

$$
\begin{aligned}
\rho_{N L, e}\left(z, z_{0}, k_{0}, K\right)= & \frac{k_{0}^{3}}{4 \pi^{3} c}\left(\varepsilon_{i}^{\prime}\left|g_{e e}^{11}\right|^{2}+\mu_{i}^{\prime}\left|g_{e m}^{1 K}\right|^{2}+\mu_{i}^{\prime}\left|g_{e m}^{1 z}\right|^{2}+\mu_{i}^{\prime}\left|g_{e m}^{K 1}\right|^{2}\right. \\
& \left.+\varepsilon_{i}^{\prime}\left|g_{e e}^{K K}\right|^{2}+\varepsilon_{i}^{\prime}\left|g_{e e}^{K z}\right|^{2}+\mu_{i}^{\prime}\left|g_{e m}^{z 1}\right|^{2}+\varepsilon_{i}^{\prime}\left|g_{e e}^{z K}\right|^{2}+\varepsilon_{i}^{\prime}\left|g_{e e}^{z z}\right|^{2}\right),
\end{aligned}
$$

where $\varepsilon_{i}^{\prime}=\varepsilon_{i}\left(z_{0}\right)$ and $\mu_{i}^{\prime}=\mu_{i}\left(z_{0}\right)$ are the imaginary parts of the relative permittivity and permeability at the source coordinate [20]. Note that all the $g_{e m}$ exchange terms vanish if $\mu_{i}=0$, which is the case in most materials used in optoelectronic devices. The magnetic NLDOS is similarly given by

$$
\begin{aligned}
\rho_{N L, m}\left(z, z_{0}, k_{0}, K\right)= & \frac{k_{0}^{3}}{4 \pi^{3} c}\left(\mu_{i}^{\prime}\left|g_{m m}^{11}\right|^{2}+\varepsilon_{i}^{\prime}\left|g_{m e}^{1 K}\right|^{2}+\varepsilon_{i}^{\prime}\left|g_{m e}^{1 z}\right|^{2}+\varepsilon_{i}^{\prime}\left|g_{m e}^{K 1}\right|^{2}\right. \\
& \left.+\mu_{i}^{\prime}\left|g_{m m}^{K K}\right|^{2}+\mu_{i}^{\prime}\left|g_{m m}^{K z}\right|^{2}+\varepsilon_{i}^{\prime}\left|g_{m e}^{z 1}\right|^{2}+\mu_{i}^{\prime}\left|g_{m m}^{z K}\right|^{2}+\mu_{i}^{\prime}\left|g_{m m}^{z z}\right|^{2}\right) .
\end{aligned}
$$

As with the LDOS, the total electromagnetic NLDOS can be calculated as $\rho_{N L, \text { tot }}=1 / 2\left(|\varepsilon| \rho_{N L, e}+|\mu| \rho_{N L, m}\right)$. To calculate the NLDOS, the dyadic components need to be calculated from the optical admittances for a chosen source point $z_{0}$ as specified in Appendix A, but they do not simplify as much as the LDOS in Eqs. (28) and (29).

\section{E. Application to fluctuational electrodynamics}

Having access to the dyadic Green's function and consequently the LDOS and NLDOS enables directly formulating the classical or quantized FED. In both, $\mathbf{J}_{e}$ and $\mathbf{J}_{m}$ are written as thermally fluctuating source currents, which depend on the position through the local temperature and/or excitation of the carrier populations. To finally obtain the position-dependent photon numbers in FED, the NLDOS functions have to be multiplied with the fluctuating source terms, then integrated over all the source coordinates, and finally normalized with the LDOS. Specifically in QFED, $\mathbf{J}_{e}$ and $\mathbf{J}_{m}$ are represented by polarization and magnetization noise current operators, which are defined in terms of the bosonic source-field operators and the imaginary parts of the relative permittivity and permeability [18-20]. In QFED, the photon-number expectation values at coordinate $z$ can therefore be calculated with the help of the LDOS and NLDOS as

$$
\left\langle\hat{n}_{e / m / \mathrm{tot}}\left(z, K, k_{0}\right)\right\rangle=\frac{\int \rho_{N L, e / m / \mathrm{tot}}\left(z, z_{0}, k_{0}, K\right)\left\langle\hat{\eta}\left(z_{0}, k_{0}\right)\right\rangle d z_{0}}{\rho_{e / m / \mathrm{tot}}\left(z, k_{0}, K\right)},
$$

where $\hat{\eta}$ is the source-field photon-number operator, whose expectation value follows the Bose-Einstein distribution for purely thermal fields or electrically excited semiconductors, if the material's Fermi-level separation is included as the photon chemical potential [32]. Finally, the local net emission rate according to QFED can be calculated using the expectation values mentioned above as

$$
\begin{aligned}
\langle\hat{Q}(z)\rangle_{\omega, K}= & \hbar \omega^{2} \varepsilon_{i} \rho_{e}\left(z, k_{0}, K\right)\left[\left\langle\hat{\eta}\left(z, k_{0}\right)\right\rangle-\left\langle\hat{n}_{e}\left(z, k_{0}, K\right)\right\rangle\right] \\
& +\hbar \omega^{2} \mu_{i} \rho_{m}\left(z, k_{0}, K\right)\left[\left\langle\hat{\eta}\left(z, k_{0}\right)\right\rangle-\left\langle\hat{n}_{m}\left(z, k_{0}, K\right)\right\rangle\right],
\end{aligned}
$$

where the subindices $\omega$ and $K$ denote the variables one has to integrate over to get the total net emission rate. Note that both nonzero $\varepsilon_{i}$ and/or $\mu_{i}$ and a nonzero LDOS are needed to obtain a nonzero net emission. The dyadic Green's function components can also be used to express the damping and scattering coefficients for the interference-exact radiative transfer (IFRT) model, which is being developed to provide a computationally efficient access to the local photon numbers in Eq. (33) and consequently, to a direct integration with the chosen carrier dynamics model [20]. Once this is done, the self-consistent coupling of optical and electrical processes can be done by, e.g., coupling the IFRT model and the driftdiffusion (DD) model for carrier dynamics in a similar way as in Ref. [33], where the conventional radiative transfer model was still used instead of the IFRT. Essentially this involves evaluating $\left\langle\hat{\eta}\left(z, k_{0}\right)\right\rangle$ using the quasi-Fermi levels from DD and setting it as the source term in the IFRT model. Correspondingly, the spatial net recombination and generation rate is evaluated using Eq. (33) and the photon numbers from IFRT, and it is used as an input to the DD model. 
To comment shortly on the computational efficiency of the optical admittance method, we note that the potential benefits of the method would mostly follow from the more straightforward integration of the model with the charge transport framework and the availability of simple analytic solutions. For the full Green's functions and the related quantities, on the other hand, the calculation time is fully determined by the time required for spatial numerical integration [see, e.g., Eqs. (23) and (24)]. That, in turn, is dependent on the number of points required for the spatial grid. We leave a more detailed comparison of the computational efficiency between the presented method and, e.g., the transfer-matrix method to a possible future work on the topic.

\section{RESULTS \& DISCUSSION}

To demonstrate how the optical admittance method is used, we calculate the LDOS and NLDOS and related quantities for selected optoelectronic devices. We begin by showing how the position of the emitting quantum well (QW) affects the photon numbers in a GaN-based multi-quantum-well (MQW) flip-chip LED. Then we apply the method to map the optical confinement of a vertical cavity surface-emitting laser (VCSEL), another frequently used device architecture in modern optoelectronics. Finally in Appendix B, to show how optical admittances are applied to model graded structures, we simulate photon transport in top-emitting GaN LEDs, with and without graded antireflective coatings. The purpose of this section is to give an overview on how the information normally calculated with more complex methods can be obtained using the proposed optical admittance method. In these results we do not go notably beyond calculating the LDOS and NLDOS, but we note that they are the quantities that are needed for building fully self-consistent optoelectronic models through the use of Eqs. (32) and (33) or the interferenceexact radiative transfer model [20]. To choose a representative but sufficiently simple quantity that illustrates the optical fields created by point sources, we show normalized total photon-number expectation values defined as

$$
\mathcal{N}\left(z, z_{0}, k_{0}, K\right)=\frac{1}{\mathcal{N}_{m}} \frac{\rho_{N L, \text { tot }}\left(z, z_{0}, k_{0}, K\right)}{\rho_{\text {tot }}\left(z, k_{0}, K\right)},
$$

where $\mathcal{N}_{m}$ is the maximum of $\rho_{N L \text {,tot }} / \rho_{\text {tot }}$, limiting $\mathcal{N}$ between 0 and 1 for convenience. Comparing with Eq. (32), $\mathcal{N}$ essentially denotes the normalized photon number of the electromagnetic field created by a point source located at the chosen $z=z_{0}$. All the calculations are performed for fixed photon energies (and thereby $k_{0}$ ) and $z_{0}$, and all the results are shown as color maps, with $z$ and $K$ as the coordinate axes.

\section{A. GaN MQW flip-chip LED structure}

GaN MQW LEDs form the backbone of today's solidstate lighting technologies. Here we illustrate the optical characteristics of a flip-chip GaN LED consisting of a native GaN substrate, an InGaN/GaN MQW layer with five 3-nm-thick QWs separated by 10-nm-thick GaN barriers, a $p$-type $\mathrm{GaN}$ layer, and a thin silver contact of $20 \mathrm{~nm}$ before the interface with air. The silver contact is similar to the one in Ref. [19], where it was used to support plasmons.
We use the same thickness here for comparison, even if we do not focus on plasmon resonances in this paper. In addition, to make a simple comparison between two slightly differing structures, we place the MQW either 300 or $600 \mathrm{~nm}$ below the top contact. (These distances are chosen arbitrarily to demonstrate the method without a specific relation to the wavelength.) The calculation is made with the photon energy $E_{p}=\hbar c k_{0}$ fixed to $2.786 \mathrm{eV}, \mu$ equal to 1 everywhere, and the refractive indices $N=\sqrt{\varepsilon \mu}$ set to $2.51+0.0029 i(\mathrm{GaN})$, $2.51+0.094 i(\mathrm{InGaN}), 0.013+3.119 i$ (silver), and 1 (air), following Ref. [19].

To show how the MQW position affects the photon energy density resulting from emission in the QWs, Fig. 2 shows $\mathcal{N}$ as calculated from the optical admittances. Figures 2(a) and 2(b) show the results for the structure where the MQW is located $300 \mathrm{~nm}$ below the top contact, and the source is in the middle of the (a) lowest and (b) uppermost QW. In Figs. 2(c) and 2(d), the MQW is located $600 \mathrm{~nm}$ below the top contact, and the source is correspondingly in the (c) lowest and (d) uppermost QW. The figures are plotted on a logarithmic scale due to the large variations in $\mathcal{N}$ within the studied $z$ and $K$ range. It can be seen that as expected, the structure creates freely propagating modes in the GaN escape cone (left side of the figures up to $K / k_{0}=2.51$ ) and in the air escape cone (right side up to $K / k_{0}=1$ ), while the fields are evanescent elsewhere. Considering freely propagating modes in air (values $K / k_{0}<1$ in air), neither the MQW position nor the source position seems to substantially affect the value of $\mathcal{N}$ on the logarithmic scale. This can be expected, since the silver film reflectivity does not depend on the MQW position, and there is no mirror on the left that would cause interference effects with the field initially emitted towards the left. In addition, the $\mathcal{N}$ value is small in air due to reflection from the metal contact.

Considering emission into the GaN substrate on the left, $\mathcal{N}$ varies over roughly 2 orders of magnitude in the left side of Figs. 2(a)-2(d) due to interference with the field reflected from the silver contact. Therefore the position of the MQW region and the source point have a considerable effect on the emission towards the left. More specifically, the cases shown in Figs. 2(b) and 2(c) give larger $\mathcal{N}$ values in $\mathrm{GaN}$ in the left side at $K / k_{0}<1$ (rectangle in the bottom left corner) than those shown in Figs. 2(a) and 2(d). This could be important information, if one would need accurate angular control of the emission pattern towards the bottom side. Even in a typical flip-chip LED, this might be useful information for optimizing the total emission towards the bottom interface, where light is typically extracted through a roughened surface. In any case, based on the results of Fig. 2, the proposed model provides direct access to the emission patterns of QWs embedded in fairly complex heterostructure devices, such as the chosen flip-chip MQW LED.

\section{B. GaN VCSEL structure}

As a second short example of using the proposed optical admittance method, we study it in one of the GaN vertical cavity surface-emitting laser (VCSEL) structures reported in Ref. [34]. The structure chosen here has a 42-period distributed Bragg reflector (DBR) consisting of GaN and AlGaN 


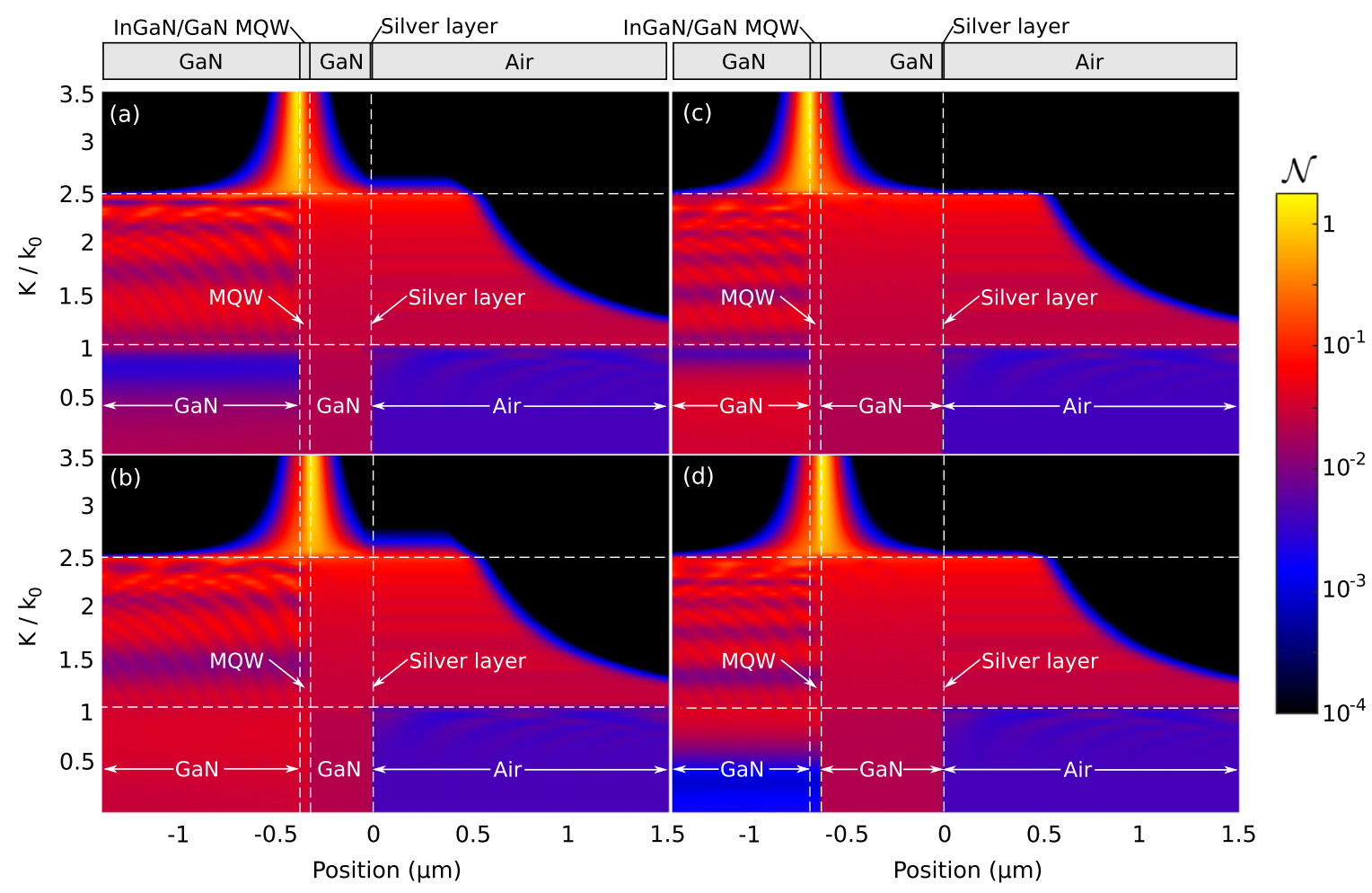

FIG. 2. 10-base logarithm of the normalized photon number $\mathcal{N}$ in the different GaN MQW flip-chip for (a) MQW located $300 \mathrm{~nm}$ below the top contact with the source in the middle of the lowest QW, (b) MQW located $300 \mathrm{~nm}$ below the top contact with the source in the middle of the uppermost QW, (c) MQW located $600 \mathrm{~nm}$ below the top contact with the source in the lowest QW, and (d) MQW located $600 \mathrm{~nm}$ below the top contact with the source in the uppermost QW. The horizontal dashed lines mark the escape cones of air and GaN, and the vertical dashed lines the position of the MQW and the metal-air interface. The photon energy and wavelength in the calculations are $2.786 \mathrm{eV}$ and $446 \mathrm{~nm}$, respectively.

in the bottom side and a seven-period DBR consisting of $\mathrm{TiO}_{2}$ and $\mathrm{SiO}_{2}$ in the top side, and a MQW layer consisting of three InGaN QWs separated by InGaN barriers. The refractive indices are obtained from Refs. [34] and [35], and the photon energy is $2.96 \mathrm{eV}$. To see how well the DBRs confine photons in the active region of the VCSEL, in Fig. 3 we plot again $\mathcal{N}$ with the source placed in the middle of the (a) first, (b) second, and (c) third QW. Also here we plot the results on a logarithmic scale due to the large variations in $\mathcal{N}$ within the studied $z$ and $K$ range.

All the cases considered in Fig. 3 show that the optical admittance method predicts strong optical confinement in the active layer of the VCSEL roughly up to $K / k_{0}=0.5$, as expected due to the DBRs. Interestingly, based on Figs. 3(a)3 (c), the quantitative details of $\mathcal{N}$ at the bottom DBR depend somewhat on the position of the light emitter. On the other

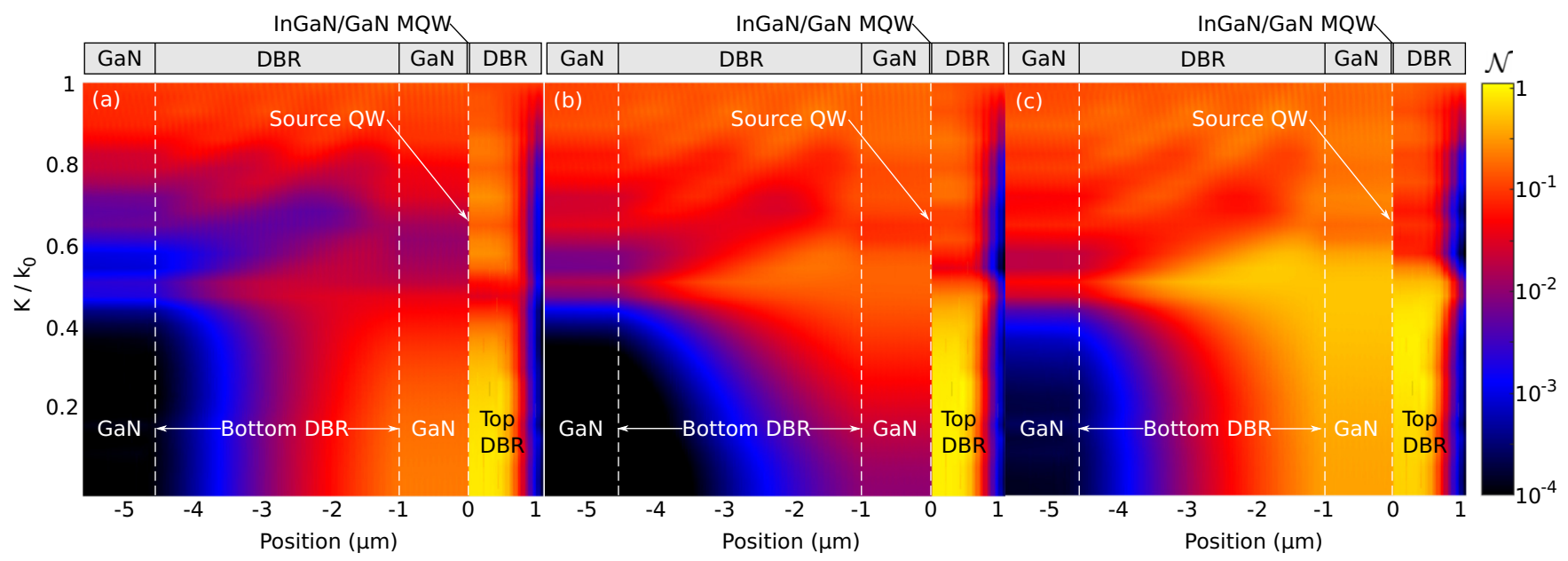

FIG. 3. 10-base logarithm of the normalized photon number $\mathcal{N}$ in the structure of Ref. [34] with the source point in the middle of the (a) first, (b) second, and (c) third QW. The photon energy and wavelength in the calculations are $2.96 \mathrm{eV}$ and $420 \mathrm{~nm}$, respectively. 
hand, the emission pattern towards the top side where light is extracted (on the right side of the figures) is roughly similar in all three cases up to $K / k_{0}=0.5$ with the chosen logarithmic scale. Overall, these results demonstrate that the optical admittances allow calculating the detailed emission patterns directly even in the complex VCSEL structures, which include DBRs consisting of tens of subsequent extremely thin layers as well as large variations in the emitted electromagnetic fields.

\section{CONCLUSIONS}

In order to realize an accurate and generally applicable model of photon transport in arbitrary lossy resonator structures, we formulated the Green's functions and the related optical quantities required for the recently introduced quantized fluctuational electrodynamics (QFED) framework using the so called optical admittance functions. The optical admittance functions can be expressed analytically for arbitrary piecewise-homogeneous layer structures and solved numerically for any embedded graded layers. In addition, the optical admittance functions enable straightforward expressions for all the local and nonlocal densities of states required for accurate modeling of light-matter interaction. Through selected examples we illustrated that by using the presented framework, one can make straightforward yet detailed analyses of energy densities and transport in a wide range of planar photonic devices, especially once the method is coupled with full-device models including electrical and thermal transport. We believe that having a directly workable self-consistent model of electrical and optical processes will enable both understanding the physics of planar devices better, as well as developing new photonic device functionalities for thin-film structures.

\section{ACKNOWLEDGMENTS}

We acknowledge financial support from the Academy of Finland and the European Research Council (Horizon 2020 Research and Innovation Programme, Grant Agreement No. 638173).

\section{APPENDIX A: DYADIC GREEN'S FUNCTIONS FROM OPTICAL ADMITTANCES}

To make the presentation of the spectral dyadic components more clear, we define integral terms related to TE optical admittances as

$$
\begin{aligned}
& \mathcal{Z}^{T E}\left(z_{1}, z_{2}, \gamma^{T E}\right) \\
& \quad=\exp \left(-i k_{0} \int_{z_{1}}^{z_{2}} \mu \gamma^{T E}\left(z^{\prime}\right) d z^{\prime}\right) \Theta\left(z_{2}-z_{1}\right),
\end{aligned}
$$

where $z_{1}$ and $z_{2}$ are position coordinates and $\gamma^{T E}$ is the TE optical admittance calculated for a right- or leftward mode. For the same purpose, we define similar integral terms for TM optical admittances as

$$
\begin{aligned}
& \mathcal{Z}^{T M}\left(z_{1}, z_{2}, \gamma^{T M}\right) \\
& \quad=\exp \left(-i k_{0} \int_{z_{1}}^{z_{2}} \varepsilon \gamma^{T M}\left(z^{\prime}\right) d z^{\prime}\right) \Theta\left(z_{2}-z_{1}\right) .
\end{aligned}
$$

The coordinates are defined as in the article so that $\mathbf{u}_{1}$ is the direction of the electric (magnetic) field in the TE (TM) modes and $\mathbf{u}_{K}$ is the direction perpendicular to $\mathbf{u}_{1}$ in the $x y$ plane, so that $\mathbf{u}_{1}=\mathbf{u}_{K} \times \mathbf{u}_{z}$. The dyadic Green's function components are derived following Ref. [20], making use of the reciprocity relations $G^{T E}\left(z, z_{0}, k_{0}, K\right)=G^{T E}\left(z_{0}, z, k_{0}, K\right)$ and $G^{T M}\left(z, z_{0}, k_{0}, K\right)=G^{T M}\left(z_{0}, z, k_{0}, K\right)$.

\section{Electric field from electric sources}

Using the dyadic Green's function, the electric field created by an electric source is obtained from the relation

$$
\begin{aligned}
E\left(z, k_{0}, K\right) & =\int_{-\infty}^{\infty} \overleftrightarrow{G} e e\left(z, z_{0}, k_{0}, K\right) \cdot \mathbf{J}_{\text {eff }}^{e}\left(z_{0}, k_{0}, K\right) d z_{0} \\
& =\int_{-\infty}^{\infty}\left[\begin{array}{ccc}
g_{e e}^{11}\left(z, z_{0}, k_{0}, K\right) & 0 & 0 \\
0 & g_{e e}^{K K}\left(z, z_{0}, k_{0}, K\right) & g_{e e}^{K z}\left(z, z_{0}, k_{0}, K\right) \\
0 & g_{e e}^{z K}\left(z, z_{0}, k_{0}, K\right) & g_{e e}^{z z}\left(z, z_{0}, k_{0}, K\right)
\end{array}\right] \cdot\left[\begin{array}{c}
J_{\text {eff }, 1}^{E}\left(z_{0}, k_{0}, K\right) \\
J_{\text {eff, } \mathrm{K}}^{E}\left(z_{0}, k_{0}, K\right) \\
J_{\text {eff }, \mathrm{z}}^{E}\left(z_{0}, k_{0}, K\right)
\end{array}\right] d z_{0},
\end{aligned}
$$

with the elements (separated into components associated with the TE and TM polarization) given below.

$$
\text { a. } T E
$$

$$
\begin{gathered}
g_{e e}^{11}\left(z, z_{0}, k_{0}, K\right)=G^{T E}=-\frac{i}{k_{0}\left[\gamma_{r}^{T E}(z)+\gamma_{l}^{T E}(z)\right]} \quad\left[\mathcal{Z}^{T E}\left(z_{0}, z, \gamma_{l}^{T E}\right)+\mathcal{Z}^{T E}\left(z, z_{0}, \gamma_{r}^{T E}\right)\right] . \\
\text { b. } \mathbf{T M}
\end{gathered}
$$

$$
\begin{aligned}
g_{e e}^{K K}\left(z, z_{0}, k_{0}, K\right)= & \frac{1}{\varepsilon(z) \varepsilon\left(z_{0}\right)} \frac{1}{k_{0}^{2}} \frac{\partial^{2}}{\partial z \partial z_{0}} G^{T M}-\frac{1}{\varepsilon\left(z_{0}\right) k_{0}^{2}} \delta\left(z-z_{0}\right)=-\frac{i}{k_{0}\left[\gamma_{r}^{T M}\left(z_{0}\right)+\gamma_{l}^{T M}\left(z_{0}\right)\right]} \\
& \times\left[\gamma_{l}^{T M}\left(z_{0}\right) \gamma_{r}^{T M}(z) \mathcal{Z}^{T M}\left(z_{0}, z, \gamma_{r}^{T M}\right)+\gamma_{r}^{T M}\left(z_{0}\right) \gamma_{l}^{T M}(z) \mathcal{Z}^{T M}\left(z, z_{0}, \gamma_{l}^{T M}\right)\right]
\end{aligned}
$$




$$
\begin{aligned}
& g_{e e}^{z K}\left(z, z_{0}, k_{0}, K\right)=-\frac{i K}{\varepsilon\left(z_{0}\right) \varepsilon(z) k_{0}^{2}} \frac{\partial}{\partial z_{0}} G^{T M}=-\frac{K / k_{0}^{2}}{\varepsilon(z)} \frac{i}{\gamma_{l}^{T M}\left(z_{0}\right)+\gamma_{r}^{T M}\left(z_{0}\right)} \\
& \times\left[\gamma_{l}^{T M}\left(z_{0}\right) \mathcal{Z}^{T M}\left(z_{0}, z, \gamma_{r}^{T M}\right)-\gamma_{r}^{T M}\left(z_{0}\right) \mathcal{Z}^{T M}\left(z, z_{0}, \gamma_{l}^{T M}\right)\right] . \\
& g_{e e}^{K z}\left(z, z_{0}, k_{0}, K\right)= \frac{i K}{\varepsilon(z) \varepsilon\left(z_{0}\right) k_{0}^{2}} \frac{\partial}{\partial z} G^{T M}=\frac{i K}{k_{0}^{2}} \frac{1}{\varepsilon\left(z_{0}\right)} \frac{1}{\gamma_{l}^{T M}(z)+\gamma_{r}^{T M}(z)} \\
& \times\left[\gamma_{l}^{T M}(z) \mathcal{Z}^{T M}\left(z, z_{0}, \gamma_{r}^{T M}\right)-\gamma_{r}^{T M}(z) \mathcal{Z}^{T M}\left(z_{0}, z, \gamma_{l}^{T M}\right)\right] . \\
& g_{e e}^{z z}\left(z, z_{0}, k_{0}, K\right)= \frac{K^{2}}{\varepsilon\left(z_{0}\right) \varepsilon(z) k_{0}^{2}} G^{T M}-\frac{1}{\varepsilon\left(z_{0}\right) k_{0}^{2}} \delta\left(z-z_{0}\right) \\
&=-\frac{K^{2} / k_{0}^{3}}{\varepsilon\left(z_{0}\right) \varepsilon(z)} \frac{i}{\left(\gamma_{r}^{T M}\left(z_{0}\right)+\gamma_{l}^{T M}\left(z_{0}\right)\right)}\left[\mathcal{Z}^{T M}\left(z, z_{0}, \gamma_{l}^{T M}\right)+\mathcal{Z}^{T M}\left(z_{0}, z, \gamma_{r}^{T M}\right)\right]-\frac{1}{\varepsilon\left(z_{0}\right) k_{0}^{2}} \delta\left(z-z_{0}\right) .
\end{aligned}
$$

\section{Magnetic field from electric sources}

The magnetic field related to an electric source is obtained when the Green's dyadic $\overleftrightarrow{G}^{e e}$ in Eq. (A3) is replaced by $\overleftrightarrow{G}^{m e}$ with the following elements.

$$
\begin{gathered}
g_{m e}^{K 1}\left(z, z_{0}, k_{0}, K\right)=\frac{1}{k_{0} \mu(z)} \frac{\partial}{\partial z} G^{T E}=\frac{1}{k_{0}\left(\gamma_{l}^{T E}(z)+\gamma_{r}^{T E}(z)\right)}\left[\gamma_{l}^{T E}(z) \mathcal{Z}^{T E}\left(z, z_{0}, \gamma_{r}^{T E}\right)-\gamma_{r}^{T E}(z) \mathcal{Z}^{T E}\left(z_{0}, z, \gamma_{l}^{T E}\right)\right] . \\
g_{m e}^{z 1}\left(z, z_{0}, k_{0}, K\right)=-\frac{1}{\mu(z)} \frac{i K}{k_{0}} G^{T E}=-\frac{1}{\mu(z)} \frac{K}{k_{0}^{2}} \frac{1}{\gamma_{r}^{T E}(z)+\gamma_{l}^{T E}(z)}\left[\mathcal{Z}^{T E}\left(z_{0}, z, \gamma_{l}^{T E}\right)+\mathcal{Z}^{T E}\left(z, z_{0}, \gamma_{r}^{T E}\right)\right] .
\end{gathered}
$$

\section{b. $T M$}

$$
\begin{gathered}
g_{m e}^{1 K}\left(z, z_{0}, k_{0}, K\right)=\frac{1}{k_{0} \varepsilon\left(z_{0}\right)} \frac{\partial}{\partial z_{0}} G^{T M}=\frac{1}{k_{0}\left[\gamma_{l}^{T M}\left(z_{0}\right)+\gamma_{r}^{T M}\left(z_{0}\right)\right]}\left[\gamma_{l}^{T M}\left(z_{0}\right) \mathcal{Z}^{T M}\left(z_{0}, z, \gamma_{r}^{T M}\right)-\gamma_{r}^{T M}\left(z_{0}\right) \mathcal{Z}^{T M}\left(z, z_{0}, \gamma_{l}^{T M}\right)\right] . \\
g_{m e}^{1 z}\left(z, z_{0}, k_{0}, K\right)=\frac{i K}{k_{0}} \frac{1}{\varepsilon\left(z_{0}\right)} G^{T M}=\frac{1}{\varepsilon\left(z_{0}\right)} \frac{K}{k_{0}^{2}} \frac{1}{\gamma_{r}^{T M}\left(z_{0}\right)+\gamma_{l}^{T M}\left(z_{0}\right)}\left[\mathcal{Z}^{T M}\left(z, z_{0}, \gamma_{l}^{T M}\right)+\mathcal{Z}^{T M}\left(z_{0}, z, \gamma_{r}^{T M}\right)\right] .
\end{gathered}
$$

\section{Magnetic field from magnetic sources}

The magnetic field related to a magnetic source is obtained when the electric current $\mathbf{J}_{\text {eff }}^{e}$ in Eq. (A3) is replaced by the magnetic current $\mathbf{J}_{\text {eff }}^{m}$, and the Green's dyadic $\overleftrightarrow{G}^{e e}$ is replaced by $\overleftrightarrow{G}^{m m}$ with the following elements.

$$
\begin{aligned}
g_{m m}^{K K}\left(z, z_{0}, k_{0}, K\right)= & \frac{1}{\mu\left(z_{0}\right) \mu(z)} \frac{1}{k_{0}^{2}} \frac{\partial^{2}}{\partial z \partial z_{0}} G^{T M}-\frac{1}{\mu\left(z_{0}\right) k_{0}^{2}} \delta\left(z-z_{0}\right)=-\frac{i}{k_{0}\left[\gamma_{r}^{T E}\left(z_{0}\right)+\gamma_{l}^{T E}\left(z_{0}\right)\right]} \\
& \times\left[\gamma_{l}^{T E}\left(z_{0}\right) \gamma_{r}^{T E}(z) \mathcal{Z}^{T E}\left(z_{0}, z, \gamma_{r}^{T E}\right)+\gamma_{r}^{T E}\left(z_{0}\right) \gamma_{l}^{T E}(z) \mathcal{Z}^{T E}\left(z, z_{0}, \gamma_{l}^{T E}\right)\right] . \\
g_{m m}^{z K}\left(z, z_{0}, k_{0}, K\right)= & -\frac{i K}{\mu\left(z_{0}\right) \mu(z) k_{0}^{2}} \frac{\partial}{\partial z_{0}} G^{T E}=-\frac{K / k_{0}^{2}}{\mu(z)} \frac{i}{\left(\gamma_{l}^{T E}\left(z_{0}\right)+\gamma_{r}^{T E}\left(z_{0}\right)\right)} \\
& \times\left[\gamma_{l}^{T E}\left(z_{0}\right) \mathcal{Z}^{T E}\left(z_{0}, z, \gamma_{r}^{T E}\right)-\gamma_{r}^{T E}\left(z_{0}\right) \mathcal{Z}^{T E}\left(z, z_{0}, \gamma_{l}^{T E}\right)\right] . \\
g_{m m}^{K z}\left(z, z_{0}, k_{0}, K\right)= & \frac{i K}{\mu\left(z_{0}\right) \mu(z) k_{0}^{2}} \frac{\partial}{\partial z} G^{T E}=\frac{i K}{k_{0}^{2}} \frac{1}{\mu\left(z_{0}\right)} \frac{1}{\gamma_{l}^{T E}(z)+\gamma_{r}^{T E}(z)} \\
& \times\left[\gamma_{l}^{T E}(z) \mathcal{Z}^{T E}\left(z, z_{0}, \gamma_{r}^{T E}\right)-\gamma_{r}^{T E}(z) \mathcal{Z}^{T E}\left(z_{0}, z, \gamma_{l}^{T E}\right)\right] .
\end{aligned}
$$




$$
\begin{aligned}
g_{m m}^{z z}\left(z, z_{0}, k_{0}, K\right) & =\frac{K^{2}}{\mu\left(z_{0}\right) \mu(z) k_{0}^{2}} G^{T E}-\frac{1}{\mu\left(z_{0}\right) k_{0}^{2}} \delta\left(z-z_{0}\right) \\
& =-\frac{K^{2} / k_{0}^{3}}{\mu\left(z_{0}\right) \mu(z)} \frac{i}{\left[\gamma_{r}^{T E}\left(z_{0}\right)+\gamma_{l}^{T E}\left(z_{0}\right)\right]}\left[\mathcal{Z}^{T E}\left(z, z_{0}, \gamma_{l}^{T E}\right)+\mathcal{Z}^{T E}\left(z_{0}, z, \gamma_{r}^{T E}\right)\right]-\frac{1}{\mu\left(z_{0}\right) k_{0}^{2}} \delta\left(z-z_{0}\right)
\end{aligned}
$$

$$
g_{m m}^{11}\left(z, z_{0}, k_{0}, K\right)=G^{T M}=-\frac{i}{k_{0}\left[\gamma_{r}^{T M}(z)+\gamma_{l}^{T M}(z)\right]}\left[\mathcal{Z}^{T M}\left(z_{0}, z, \gamma_{l}^{T M}\right)+\mathcal{Z}^{T M}\left(z, z_{0}, \gamma_{r}^{T M}\right)\right]
$$

\section{Electric field from magnetic sources}

The electric field related to a magnetic source is obtained when the electric current $\mathbf{J}_{\text {eff }}^{e}$ in Eq. (A3) is replaced by the magnetic current $\mathbf{J}_{\text {eff }}^{m}$, and the Green's dyadic $\overleftrightarrow{G}^{e e}$ is replaced by $\overleftrightarrow{G}^{e m}$ with the following elements.

a. $T E$

$$
\begin{aligned}
g_{\text {em }}^{1 K}\left(z, z_{0}, k_{0}, K\right)= & -\frac{1}{k_{0} \mu\left(z_{0}\right)} \frac{\partial}{\partial z_{0}} G^{T E}=-\frac{1}{k_{0}\left(\gamma_{l}^{T E}\left(z_{0}\right)+\gamma_{r}^{T E}\left(z_{0}\right)\right)} \\
& \times\left[\gamma_{l}^{T E}\left(z_{0}\right) \mathcal{Z}^{T E}\left(z_{0}, z, \gamma_{r}^{T E}\right)-\gamma_{r}^{T E}\left(z_{0}\right) \mathcal{Z}^{T E}\left(z, z_{0}, \gamma_{l}^{T E}\right)\right] . \\
g_{\text {em }}^{1 z}\left(z, z_{0}, k_{0}, K\right)=-\frac{i K}{k_{0} \mu\left(z_{0}\right)} G^{T E}= & -\frac{1}{\mu\left(z_{0}\right)} \frac{K}{k_{0}^{2}} \frac{1}{\gamma_{r}^{T E}\left(z_{0}\right)+\gamma_{l}^{T E}\left(z_{0}\right)}\left[\mathcal{Z}^{T E}\left(z, z_{0}, \gamma_{l}^{T E}\right)+\mathcal{Z}^{T E}\left(z_{0}, z, \gamma_{r}^{T E}\right)\right] .
\end{aligned}
$$

$$
\begin{aligned}
g_{e m}^{K 1}\left(z, z_{0}, k_{0}, K\right)= & -\frac{1}{\varepsilon(z) k_{0}} \frac{\partial}{\partial z} G^{T M}=-\frac{1}{k_{0}\left[\gamma_{l}^{T M}(z)+\gamma_{r}^{T M}(z)\right]} \\
& \times\left[\gamma_{l}^{T M}(z) \mathcal{Z}^{T M}\left(z, z_{0}, \gamma_{r}^{T M}\right)-\gamma_{r}^{T M}(z) \mathcal{Z}^{T M}\left(z_{0}, z, \gamma_{l}^{T M}\right)\right] . \\
g_{e m}^{z 1}\left(z, z_{0}, k_{0}, K\right)=\frac{1}{\varepsilon(z)} \frac{i K}{k_{0}} G^{T M}= & \frac{1}{\varepsilon(z)} \frac{K}{k_{0}^{2}} \frac{1}{\gamma_{r}^{T M}(z)+\gamma_{l}^{T M}(z)}\left[\mathcal{Z}^{T M}\left(z_{0}, z, \gamma_{l}^{T M}\right)+\mathcal{Z}^{T M}\left(z, z_{0}, \gamma_{r}^{T M}\right)\right] .
\end{aligned}
$$

\section{APPENDIX B: TOP-EMITTING GAN LED WITH GRADED ITO}

To shortly demonstrate the use of optical admittances in graded-index structures, as an additional example we study the optical admittance method in top-emitting LEDs, where light is extracted through a transparent top contact instead of the roughened bottom side. In top-emitting LEDs as well as in various solar cells, antireflective (AR) coatings are often used, as they enhance the coupling of light between the semiconductor structure and free space. The use of gradedindex materials can be especially attractive, as they have been reported to decrease the reflectivity more and over a wider range of incidence angles than homogeneous quarter-wave ITO layers [36-38]. Following Ref. [37], we choose three different top-emitting LED structures, all of which again have a MQW region including five InGaN QWs with a thickness of $3 \mathrm{~nm}$, separated by $10-\mathrm{nm}$-thick GaN barriers. After the last $\mathrm{QW}$, there is a 300-nm-thick layer of $\mathrm{GaN}$ before the AR coating, which consists of either (a) $500 \mathrm{~nm}$ of graded indium tin oxide (ITO) with a refractive index $N$ varying from 2.19 to 1.17, similarly as in Ref. [37], (b) ungraded ITO with $N=1.17$, or (c) ungraded ITO with $N=2.19$. In the ungraded cases, the coating thickness is always a quarter of the wavelength in the medium. Again, the permeability is 1 everywhere, and the refractive indices for $\mathrm{GaN}$ and $\mathrm{InGaN}$ are the same as those of the flip-chip LED in the main article text. The photon energy is $2.786 \mathrm{eV}$, and the source is placed in the middle of the topmost QW.

Figure 4 shows $\mathcal{N}$ as a function of position and $K$ in the LED with (a) graded ITO, (b) ITO with $n=1.17$, and (c) ITO with $n=2.19$. The source QW is located at the 0 position, and the $K$ values have been limited to the escape cone of light towards air $\left(K<k_{0}\right)$, as none of the chosen AR coatings prevents total internal reflection at $K>k_{0}$. For Fig. 4(a), the admittances within the graded layer have been solved numerically, while for the other layers and figures they are obtained from Eq. (19) of the article text. By comparing Figs. 4(a)-4(c), we see that $\mathcal{N}$ in air is slightly larger in (a) than in (b) and (c) at almost all $K$ values. To study this 


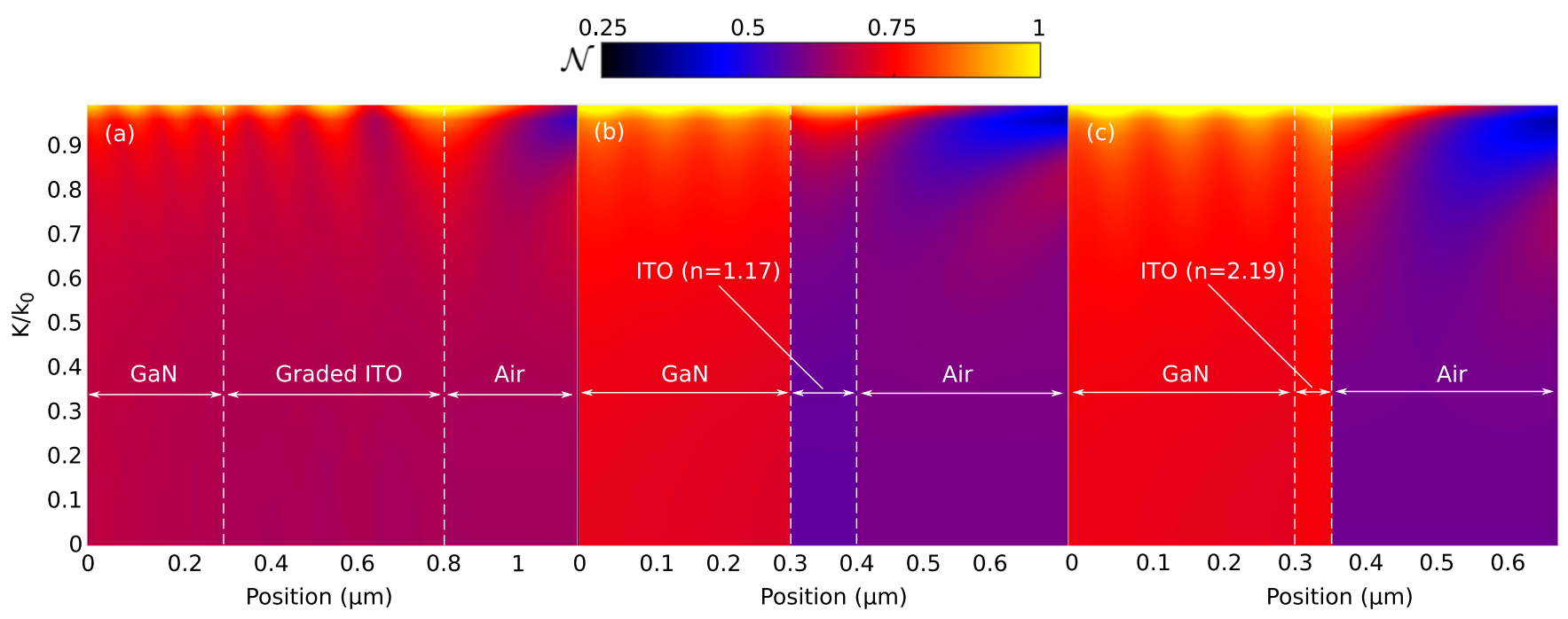

FIG. 4. Normalized photon number $\mathcal{N}$ on a linear scale in the light cone of air for a top-emitting GaN LED with different antireflective coatings: (a) $500 \mathrm{~nm}$ of graded ITO with the refractive index $N$ varying from 2.19 to 1.17 , (b) ungraded ITO with $N=1.17$, and (c) ungraded ITO with $N=2.19$. In (b) and (c) the ITO thickness is a quarter of the wavelength. The emitting QW is at the 0 position, and the different regions are marked in the figures. The photon energy and wavelength in the calculations are $2.786 \mathrm{eV}$ and $446 \mathrm{~nm}$, respectively.

further, we have calculated the reflectivities of all the AR coatings as a function of $K$ (not shown) from the optical admittances as advised in [21], and they correspond well to values reported in Ref. [37]. The nongraded AR coatings are unideal and reflect roughly $10 \%$ at normal incidence, while the graded AR coating is almost transparent. The unideal performance of the single-layer AR coatings is due to imperfect destructive interference of the reflected wave, as the ITO layer is surrounded by different materials at its two interfaces.
Based on this qualitative comparison to Ref. [37], the optical admittance method gives direct insight to experimental results of different antireflective coatings and pertinent interference effects. In addition, these results further demonstrate that the optical admittance method allows expressing the Green's functions and related quantities accurately, even in graded structures, without additional difficulties apart from solving the relatively simple differential equations (15)-(16) of the article text numerically within the graded layer.
[1] J. Cho, J. H. Park, J. K. Kim, and E. F. Schubert, White lightemitting diodes: History, progress, and future, Laser Photonics Rev. 11, 1600147 (2017).

[2] M. A. Green, Commercial progress and challenges for photovoltaics, Nat. Energy 1, 15015 (2016).

[3] J. Oksanen and J. Tulkki, Thermophotonic heat pump-A theoretical model and numerical simulations, J. Appl. Phys. 107, 093106 (2010).

[4] T. Sadi, P. Kivisaari, J. Tiira, I. Radevici, T. Haggren, and J. Oksanen, Electroluminescent cooling in intracavity light emitters: Modeling and experiments, Opt. Quantum Electron. 50, 18 (2018).

[5] P. Chaisakul, D. Marris-Morini, J. Frigerio, D. Chrastina, M.-S. Rouifed, S. Cecchi, P. Crozat, G. Isella, and L. Vivien, Integrated germanium optical interconnects on silicon substrates, Nat. Photonics 8, 482 (2014).

[6] N. Youngblood and M. Li, Integration of 2D materials on a silicon photonics platform for optoelectronics applications, Nanophotonics 6, 1205 (2017).

[7] S. Chu, W. Li, Y. Yan, T. Hamann, I. Shih, D. Wang, and Z. Mi, Roadmap on solar water splitting: Current status and future prospects, Nano Futures 1, 022001 (2017).

[8] C. Wadia, A. P. Alivisatos, and D. M. Kammen, Materials availability expands the opportunity for large-scale photovoltaics deployment, Environ. Sci. Technol. 43, 2072 (2009).
[9] K. Deisseroth, Optogenetics: 10 years of microbial opsins in neuroscience, Nat. Neurosci. 18, 1213 (2015).

[10] Y. Li, T. Wen, R. Zhao, X. Liu, T. Ji, H. Wang, X. Shi, J. Shi, J. Wei, Y. Zhao, X. Wu, and G. Nie, Localized electric field of plasmonic nanoplatform enhanced photodynamic tumor therapy, ACS Nano 8, 11529 (2014).

[11] B. Monemar, B. J. Ohlsson, N. F. Gardner, and L. Samuelson, Chapter seven-Nanowire-based visible light emitters, Present status and outlook, Semicond. Semimetals 94, 227 (2016).

[12] X. Wang, M. R. Khan, J. L. Gray, M. A. Alam, and M. S. Lundstrom, Design of GaAs solar cells operating close to the Shockley-Queisser limit, IEEE J. Photovolt. 3, 737 (2013)

[13] S. M. Durbin and J. L. Gray, Numerical modeling of photon recycling in solar cells, IEEE Trans. Electron Devices 41, 239 (1994).

[14] T. Sadi, J. Oksanen, and J. Tulkki, Effect of plasmonic losses on light emission enhancement in quantum-wells coupled to metallic gratings, J. Appl. Phys. 114, 223104 (2013).

[15] A. W. Walker, O. Höhn, D. N. Micha, B. Bläsi, A. W. Bett, and F. Dimroth, Impact of photon recycling on GaAs solar cell designs, IEEE J. Photovolt. 5, 1636 (2015).

[16] X. Wang, M. R. Khan, M. Lundstrom, and P. Bermel, Performance-limiting factors for GaAs-based single nanowire photovoltaics, Opt. Express 22, A344 (2014). 
[17] P. Kivisaari, Y. Chen, and N. Anttu, Emission enhancement, light extraction and carrier dynamics in InGaAs/GaAs nanowire arrays, Nano Futures 2, 015001 (2018).

[18] M. Partanen, T. Häyrynen, J. Tulkki, and J. Oksanen, Generalized noise terms for the quantized fluctuational electrodynamics, J. Phys. B 50, 055503 (2017).

[19] M. Partanen, T. Häyrynen, J. Tulkki, and J. Oksanen, Quantized fluctuational electrodynamics for three-dimensional plasmonic structures, Phys. Rev. A 95, 013848 (2017).

[20] M. Partanen, T. Häyrynen, and J. Oksanen, Interference-exact radiative transfer equation, Sci. Rep. 7, 11534 (2017).

[21] E. Hild and M. W. Evans, Calculation of the infrared reflection spectra of inhomogeneously doped silicon semiconductor layers at an arbitrary angle of incidence, J. Appl. Phys. 59, 1822 (1986).

[22] E. Hild, A. Deák, L. Naszályi, Ö. Sepsi, N. Ábrahám, and Z. Hórvölgyi, Use of the optical admittance function and its WKB approximation to simulate and evaluate transmittance spectra of graded-index colloidal films, J. Opt. A 9, 920 (2007).

[23] C. H. Henry, Theory of spontaneous emission noise in open resonators and its application to lasers and optical amplifiers, J. Lightwave Technol. 4, 288 (1986).

[24] T. Makino, Transfer-matrix formulation of spontaneous emission noise of DFB semiconductor lasers, J. Lightwave Technol. 9, 84 (1991).

[25] B. Tromborg, H. Olesen, X. Pan, and S. Saito, Transmission line description of optical feedback and injection locking for Fabry-Perot and DFB lasers, IEEE J. Quantum Electron. 23, 1875 (1987).

[26] J. E. Sipe, New Green-function formalism for surface optics, J. Opt. Soc. Am. B 4, 481 (1987).

[27] A. Narayanaswamy and Y. Zheng, A Green's function formalism of energy and momentum transfer in fluctuational electrodynamics, J. Quant. Spectrosc. Radiat. Transfer 132, 12 (2014).

[28] O. Di Stefano, S. Savasta, and R. Girlanda, Mode expansion and photon operators in dispersive and absorbing dielectrics, J. Mod. Opt. 48, 67 (2001).
[29] G. B. Arfken, H. J. Weber, and F. E. Harris, Mathematical Methodsfor Physicists, 7th ed. (Academic Press, Oxford, 2012).

[30] K. Joulain, R. Carminati, J.-P. Mulet, and J.-J. Greffet, Definition and measurement of the local density of electromagnetic states close to an interface, Phys. Rev. B 68, 245405 (2003).

[31] K. Joulain, J.-P. Mulet, F. Marquier, R. Carminati, and J.-J. Greffet, Surface electromagnetic waves thermally excited: Radiative heat transfer, coherence properties and Casimir properties revisited in the near field, Surf. Sci. Rep. 57, 59 (2005).

[32] P. Würfel, The chemical potential of radiation, J. Phys. C 15, 3967 (1982).

[33] J. Oksanen and J. Tulkki, Effects of photon transport, emission saturation and reflection losses on thermophotonic cooling, Proc. SPIE 7951, 79510H (2011).

[34] E. Hashemi, J. Gustavsson, J. Bengtsson, M. Stattin, G. Cosendey, N. Grandjean, and A. Haglund, Engineering the lateral optical guiding in gallium nitride-based vertical-cavity surface-emitting laser cavities to reach the lowest threshold gain, Jpn. J. Appl. Phys. 52, 08JG04 (2013).

[35] T. Siefke, S. Kroker, K. Pfeiffer, O. Puffky, K. Dietrich, D. Franta, I. Ohlídal, A. Szeghalmi, E.-B. Kley, and A. Tünnermann, Materials pushing the application limits of wire grid polarizers further into the deep ultraviolet spectral range, Adv. Opt. Mater. 4, 1780 (2016).

[36] J.-Q. Xi, M. F. Schubert, J. K. Kim, E. F. Schubert, M. Chen, S.-Y. Lin, W. Liu, and J. A. Smart, Optical thin-film materials with low refractive index for broadband elimination of Fresnel reflection, Nat. Photonics 1, 176 (2007).

[37] J. K. Kim, S. Chhajed, M. F. Schubert, E. F. Schubert, A. J. Fischer, M. H. Crawford, J. Cho, H. Kim, and C. Sone, Light-extraction enhancement of GaInN light-emitting diodes by graded-refractive-index indium tin oxide anti-reflection contact, Adv. Mater. 20, 801 (2008).

[38] C.-Y. Fang, Y.-L. Liu, Y.-C. Lee, H.-L. Chen, D.-H. Wan, and C.-C. Yu, Nanoparticle stacks with graded refractive indices enhance the omnidirectional light harvesting of solar cells and the light extraction of light-emitting diodes, Adv. Funct. Mater. 23, 1412 (2013). 